\title{
Numerical Optimization for Source-Drain Channel Resistance of AlGaN/GaN HEMTS
}

\author{
Rajab Yahyazadeh $^{1^{*}}$, Zahra Hashempour ${ }^{1}$ \\ ${ }^{1}$ Department of Physics, Khoy Branch, Islamic Azad University, Khoy, Iran P.O.Box: 175-58135
}

*Corresponding Author

DOI: https://doi.org/10.30880/jst.2019.11.01.001

Received 1 January 2019; Accepted 1 June 2019; Available online 10 June 2019

\begin{abstract}
A numerical model for the source-drain channel resistance based high electron mobility transistors has been developed that is capable to predict accurately the effects of polarization Coulomb Field Scattering (PCF), multi sub-band on source-drain channel resistance. Salient features of the model are incorporated of fully and partially occupied sub-bands in the interface quantum well, combined with a self-consistent solution of the Schrödinger and Poisson equations. In addition, to develop the model, accurate two-dimensional electron gas mobility and modified wave function in barrier AlGaN have been used. According to the numerical calculations, the effect of multi sub-band and PCF scattering on the increase of source-drain channel resistance is $35 \%$ and $65 \%$, respectively, with the effect of PCF being almost twice as high as multi sub-band. The calculated model results are in very good agreement with existing experimental data for high electron mobility transistors device.
\end{abstract}

Keywords: PCF scattering, multi sub-band, channel resistance

\section{Introduction}

GaN high electron mobility transistors (HEMTs) are used in high frequency, high power, and robust low-noise applications [1-3]. Linearity is one of the most crucial figures of merit for the application of power amplifiers. For improving the device linearity, advanced device structures or epitaxial structure engineerings, such as field plate, nonlinear polarization dielectric, double-channel, and optimized barrier or cap layer thickness, have been explored [47]. Polarization Coulomb Field (PCF) scattering, which stems from the non-uniform distribution of the polarization charges at the $\mathrm{AlGaN} / \mathrm{GaN}$ interface, is a particular scattering mechanism in AlGaN/GaN HFETs. It has been reported that PCF scattering can affect electron mobility and parasitic source access resistance $\left(R_{S}\right)$ [8-9]. Besides, III-V nitride-based HEMTs simulations show that the Polarization Coulomb Field Scattering plays a very important role in limiting the device performance, especially saturation region [10,11]. However, the high power dissipation of AlGaN/GaN HEMTs operating at large biases may result in high junction temperature and enhance the phonon scattering causing a drop in carrier mobility. This effect has been reported to be of great influence on the static current characteristics and is commonly referred to as self-heating. The evidence of such an effect is a negative slope of drain current $I_{D S}$ versus drain voltage $V_{D S}$ [12]. More recently, the source-drain channel resistance of III nitride-based HEMT has been modeled by several groups analytically, numerically or analytical-numerically [12-14]. It is important to investigate systematically the dependence of AlGaN-GaN HEMT performance on the Polarization Coulomb Field Scattering and multi sub-band with including different physical parameters. In this paper, we report the results on the effects of Polarization Coulomb Field Scattering on the parasitic and channel resistance with including multi sub-band. The channel resistance of these transistors was previously calculated without including PCF scattering [16]. In the present work, a new numerical model for total resistance is presented. That is capable of determining effects of PCF scattering and multi sub-band on the parasitic and channel resistance. This is achieved by (i) using a self-consistent solution of the Schrödinger and Poisson equations in order to obtain both two-dimensional electron gas density, wave 
function, Fermi level $\left(E_{F i}\right)$ specified relative to the bottom of a triangular well, (ii) take into account the modified wave function in the AlGaN barrier, (iii) take into account the occupancy of the various sub-bands, the intrasubband, and intersubband coupling coefficients $H_{m n}$. The fringing-field effect can be ignored in the present numerical model.

\section{Material and Method}

In order to obtain accurate values for the Fermi energy, the energies of quantized levels within the 2DEG, the occupancy of the various sub-bands, the intrasubband and intersubband coupling coefficients, potential profiles, wave function and the sheet carrier concentration for the 2DEG in AlGaN/GaN heterostructures; both the Schrödinger and Poisson equations must be solved self-consistently. This has been achieved by solving Schrödinger's equation and simultaneously taking into account the electrostatic potential obtained from Poisson's equation, as well as the image and exchange-correlation potentials using Numerov's numerical method. In the self-consistent calculation, the nonlinear formalism of the polarization-induced field as a function of $\mathrm{Al}$ mole fraction in $\mathrm{AlGaN} / \mathrm{GaN}$ heterostructures have been assumed, as well as taking into account all fully and partially occupied sub-bands within the interface 2DEG potential well $[13,15]$. Using such an approach, it is possible to calculate the 2D-electron mobility taking into account the combined contributions from each of the individual electron scattering mechanisms [16]. The field-effect transistor (FET) model used in calculations is shown schematically in Fig. 1. Knowing the electron energy in any sub-band $\left(E_{i}\right)$, the 2DEG density $\left(n_{2 D}\right)$, and the Fermi energy can be calculated [12, 16, 18, and 19]. The Quantum correction for the effective width of the 2DEG $\left(\Delta d_{2 D E G}=1 / n_{2 D} \int z n_{2 D}(z) d z\right)$ at a different electron temperature ( $\left.T_{e}\right)$ is given by [20]:

$$
\Delta d_{2 D E G}=5.6 \times 10^{-9} T_{e}+4.7 \times 10^{-8}
$$

Knowing the behavior of the 2DEG density as a function of the gate voltage, one can obtain the 2D electron mobility as a function of the gate voltage along the channel [21]. On the other hand, from the charge transport model, the channel potential is well known for $\mathrm{AlGaN} / \mathrm{GaN}$ heterostructure FETs. Hence the electron temperature can be derived from the energy balance model as [22]

$$
T_{e}=T_{0}+\left(\frac{2 e}{3 k_{B}}\right) \tau_{\text {total }} \mu_{2 D E G} F^{2}
$$

where $\tau_{\text {total }}$ is the total relaxation time, $K_{B}$ is the Boltzmann constant, $T_{0}$ is the lattice temperature, $\mu_{2 D E G}$ is the electron mobility and $F$ is the electric field along the 2DEG channel.

The additional polarization charge $(\Delta \sigma)$ in the gate region can be calculated as the following relation [23]:

$$
\Delta \sigma=\frac{e_{33}^{2}}{C_{33}} \Delta E_{z}^{A l G a N}
$$

Here, $e_{33}, C_{33}$ and $n_{2 D}$ are the piezoelectric coefficients, the elastic stiffness tensor and barrier thickness of AlGaN, respectively, $\left.\Delta E_{z}^{A l G a N}=\left(V_{G S}-V_{C H}\right)\right) / d_{A l G a N}$ is the vertical direction electric field across the AlGaN barrier layer. $\Delta E_{z}^{A l G a N}$ and $\Delta \sigma$ under the various gate-source voltage $V_{G S}$ and channel potential $V_{C H}$ are calculated using Eq. (3).The energy-dependent momentum relaxation rate $1 / \tau_{P C F}$ for PCF scattering can be written as [21,24]:

$$
\frac{1}{\tau_{P C F}}=\frac{A m^{*}}{2 \pi \hbar^{2}} \int_{0}^{\pi}\left[\frac{M_{K-K^{\prime}}}{S\left(q, T_{e}\right)}\right](1-\cos \theta) d \theta
$$

where $\mathrm{A}$ is the 2-D normalization constant that converts the scattering rate per area and $\theta$ is the scattering angle between initial state $k$ and final state $k^{\prime} . S\left(q, T_{e}\right)$ is the screening function to reflect the screening effect and $M_{K-K^{\prime}}$ is the matrix element (depend on the wave function) for the transition from initial state $k$ to the final state $k^{\prime}$ [24]. The wave function, although being very practical for most of the relevant mobility-related calculation, has the drawback of being zero in the barrier region. This is equivalent to consider the barrier as infinitely high. However, alloy scattering is a mobility-limiting mechanism intrinsically related to the penetration wave function in the barrier region. This can be overcome by using a modified Fang-Howard wave function [25]. Besides PCF scattering, the other main scattering mechanisms are polar-optical-phonon (POP) scattering, piezoelectric (PE) scattering, alloy scattering (Alloy), dislocation scattering (DIS), acoustic-phonon (AP), and interface roughness (IFR) scattering [26-29,16]. The 
momentum relaxation time $\tau_{I F R}, \tau_{D I S}, \tau_{P O P}, \tau_{A l l o y}, \tau_{A P}$ and $\tau_{P E}$ can be calculated using the pre-existing calculation formula [11,24]. By Matthiessen's rule, $R_{C}$ and $R_{D}$ can be written as:

$$
\begin{aligned}
& R_{S}=\frac{m^{*}\left(L_{G S}\right)}{n_{2 D 0} e^{2} W_{G}}\left(\frac{1}{\tau_{P C F}}+\frac{1}{\tau_{P O P}^{F}}+\frac{1}{\tau_{A P}^{F}}+\frac{1}{\tau_{I F R}^{F}}+\frac{1}{\tau_{D I S}^{F}}+\frac{1}{\tau_{\text {Alloy }}^{F}}\right) \\
& R_{S}=\frac{m^{*}\left(L_{G D}\right)}{n_{2 D 0} e^{2} W_{G}}\left(\frac{1}{\tau_{P C F}}+\frac{1}{\tau_{P O P}^{F}}+\frac{1}{\tau_{A P}^{F}}+\frac{1}{\tau_{I F R}^{F}}+\frac{1}{\tau_{D I S}^{F}}+\frac{1}{\tau_{\text {Alloy }}^{F}}\right)
\end{aligned}
$$

If $\quad L_{G S}=L_{G D}$ than $R_{S}=R_{D}$

$$
\begin{gathered}
R_{C H}=R_{G}=\frac{m^{*}\left(L_{G}\right)}{n_{2 D} e^{2} W_{G}}\left(\frac{1}{\tau_{P C F}}+\frac{1}{\tau_{P O P}^{G}}+\frac{1}{\tau_{A P}^{G}}+\frac{1}{\tau_{I F R}^{G}}+\frac{1}{\tau_{D I S}^{G}}+\frac{1}{\tau_{\text {Alloy }}^{G}}\right) \\
R_{\text {total }}=R_{G}+R_{F}+2 R_{C}, R_{F}=R_{S}+R_{D}, \quad R_{C H}=R_{G}
\end{gathered}
$$

The contact resistance $R_{C}$ is constant and $R_{S}$ is of different value during the measurement. As a result, $R_{S}$ is determined by the scattering mechanisms for the electrons in the gate-source channel [27]. In order to obtain accurate values for mobility, the nonlinear formalism of the polarization-induced field as a function of $\mathrm{Al}$ mole fraction in AlmGa1-mN/GaN HEMTs have been assumed, as well as taking into account intersubband coupling coefficients $H_{m n}=\iint d z_{1} d z_{2} \psi_{m n}\left(z_{1}\right) \psi_{m n}\left(z_{2}\right) \times \exp \left(q\left|z_{1}-z_{2}\right|\right.$ and all fully and partially-occupied sub-bands within the interface 2DEG potential well. From the definition of the drift mobility we obtain [26-28]:

$$
\mu_{2 D E G}\left(T_{e}, E\right)=\frac{e}{m^{*}}\left\langle\tau_{\text {total }}\left(T_{e}, E\right)\right\rangle(9)
$$

where $\tau_{\text {total }}$ are the total relaxation times associated with PCF scattering and the other main scattering mechanisms so that these relaxation times have been calculated using the methods described in Refs [26-29, 16]. Also, the different scattering rates can be separated into two types: (i) elastic scattering due to acoustic and piezoelectric phonons, ionized impurities and interface roughness, etc., and (ii) inelastic scattering due to polar optical phonons. In order to take into consideration all scattering mechanisms in the mobility calculation, it is necessary to include all such mechanisms in the linearized Boltzmann equation and to solve it numerically using an iterative technique [9]. It should be noted that in the linearized Boltzmann equation, $\Phi(E, T)$ is the perturbation function so that to obtain the $\Phi(E, T)$ needs to take into account the contribution of all occupied sub-bands by means of following relation [16]:

$$
\frac{1}{\Phi\left(E, T_{e}\right)}=\sum_{m} \sum_{n} \frac{n_{m}}{n_{2 D E G}} \frac{1}{\Phi_{m n}}
$$

Eq. (10) indicated that all occupied states contribute to the total mobility of the two-dimensional electron gases. This equation also shows that the contribution of each sub-band depends on its occupation number (that is relative concentration $n_{m} / n_{2 D E G}$ ) such that the most significant contribution comes from the first sub-band, which has the highest occupation number. Using such an approach, it is possible to calculate the 2D-electron mobility taking into account the combined contributions from each of the individual electron scattering mechanisms. Table 1 is the material parameters used in numerical calculation method for 2DEG (Tow Dimensional Electron Gas), 2DEM (Two Dimensional Electron Mobility) and Table 2 is the Specific polarization charge values for calculation of the PCF scattering potential $(V(x, y, z)), \Delta \sigma, M_{K-K^{\prime}}$ and $\tau_{P C F} \quad\left(\tau_{P C F}=\int\left[\tau_{P C F}(E) E\left(\partial f_{0}(E) / \partial E\right) d E\right] / \int\left[E\left(\partial f_{0}(E) / \partial E\right) d e\right]\right)$ 


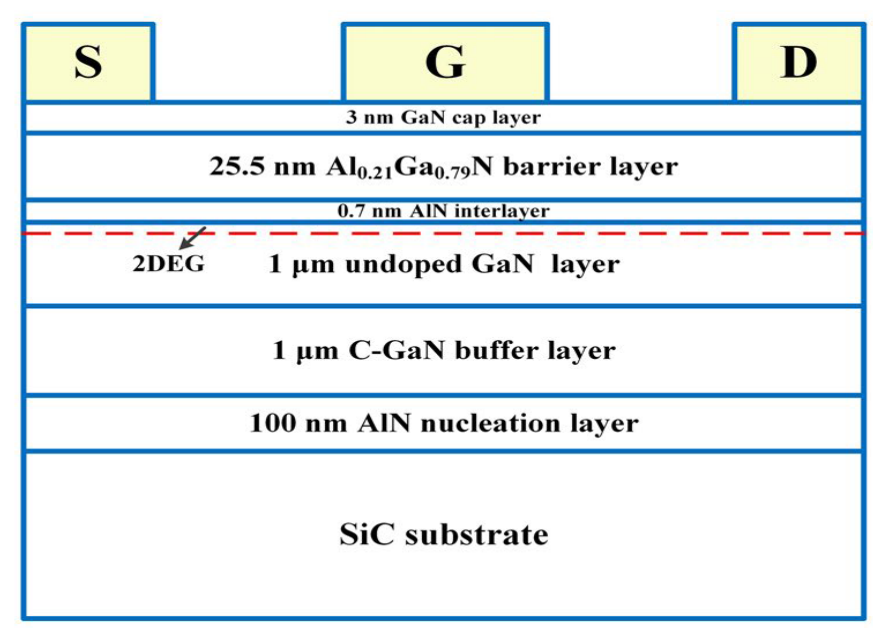

Fig. 1 - Cross-sectional view of the AlGaN/GaN HFETs

\section{Results and Discussion}

To assess the validity of the numerical model, a comparative study has been undertaken to theoretically compare the obtained results with experimental results. The material parameters used in the 2DEG, 2DEM calculations and specific polarization charge values for AlGaN/GaN HEMTs are presented in Table 1 and 2. Moreover, all other material parameters and device details have been taken from Refs. [10, 17, 20, 30 and 31] for $A l_{0.21} G a_{0.79} N / G a N$ HEMTs. Fig. 2 shows that as $L_{G}$ increases, the 2-DEG density $\left(n_{2 D}\right)$ decreases and the threshold voltage shifts towards increasingly negative values. The large values $n_{2 D}$ are attributed to the presence of polarization induced charges in AlGaN/GaN HEMTs, which have been incorporated accurately in the proposed model. The slope of the $n_{2 D}-V_{G S}$ curves corresponds to the capacitance of the structure, which is related directly to the separation between the gate and 2DEG, i.e., the thickness of the AlGaN layer. As $L_{G}$ decreases, the slope of the $n_{2 D}-V_{G S}$ curves slightly increases beyond the threshold. This is due to the fact that the gate capacitance decreases as $L_{G}$ decreases. For $L_{G}=4 \mu \mathrm{m}$, the slope is obtained as $1.9551012 \mathrm{~cm}^{-2} V^{-1}$, whereas for $L_{G}=16 \mu \mathrm{m}$, the slope decreases to $1.81012 \mathrm{~cm}^{-2} V^{-1}$. Thus, a lower value $L_{G}$ is desirable to achieve a high value of the 2-DEG density and lower values of the gate capacitance. The wave functions under different $n_{2 D}$, as shown in Fig. 3. It is apparent that the wave function is closely relevant to $n_{2 D}$. The greater $n_{2 D}$ helps the electron wave to well-function in the quantum, and the modified Fang-Howard (inset of Fig. 3) is closer to the AlGaN/GaN interface. Hence, the wave function depends on $n_{2 D}$. In Fig. 3 and 4, Calculated specific $R_{S}$ and $R_{D}$ as a function of forward gate-source voltage for PCF, POP, AP, IFR, ALLOY and DIS scatterings as well as the total $R_{S}$ (TOTAL) and the measured $R_{S}$ values at room temperature in comparison with experimental data. As shown in Fig. 5, only the resistance of PCF with the gate voltage is changed, which is related to the polarization charge $\Delta \sigma$ in the gate region. The rest of the resistors are fixed at the gate voltage, except for the resistance POP, which is extremely small in relation to the voltage and thus is not effective in the variation process of $R_{S}$ (or $R_{D}$ at this temperature. However, this will be effective at high temperatures, although investigation in this study was performed only at the $300 \mathrm{~K}$ temperature. Fig. 5 shows how this issue is corrected. The calculated $R_{\text {total }}$ for devices is shown in Fig. 5. As shown in the figure, there is a distinct difference between the experimental data and the calculated $R_{\text {total }}$ excluding the PCF scattering and the MSB effect. This means that the PCF scattering and the MSB effect are not ignorable in AlGaN/GaN HEMTs. The effect of the MSB and PCF scattering on increasing the source-drain channel resistance is almost $35 \%$ and $65 \%$, respectively. However, the increase in $V_{G S}$ and thus the decrease in the electric potential in the channel under the gate induces the decrease/increase in the negative/positive $\Delta \sigma$, according to Eq. (3). Accordingly, the PCF scattering becomes weaker with the increase in $V_{G S}$, inducing a decrease in $R_{\text {total }}$. 
Table 1- Material parameters used in 2DEG and mobility calculations for the $A l_{0.21} G a_{0.79} N / G a N$ HEMTs (Refs. 10, 14, 19-21)

\begin{tabular}{|c|c|c|}
\hline Parameters & Unit & Value \\
\hline Electron effective mass $m_{G a N}^{*}$ & $m_{0}$ & 0.228 \\
\hline Mass density & $\mathrm{kg} / \mathrm{m}^{3}$ & 6.13103 \\
\hline Static dielectric constant $\varepsilon_{S}(G a N)$ & $\varepsilon_{0}$ & 10.4 \\
\hline Optical dielectric constant $\varepsilon_{\infty}$ & $\varepsilon_{0}$ & 5.35 \\
\hline LO-phonon energy & $m e V$ & 90.5 \\
\hline Acoustic-phonon velocity & $m / s$ & $6.6 \times 10^{3}$ \\
\hline Piezoelectric constant $h_{14}$ & $V / m$ & $4.28 \times 10^{9}$ \\
\hline Deformation potential & $e V$ & 8.5 \\
\hline Elastic constant $C_{L}$ & $N / m^{2}$ & $2.66 \times 10^{11}$ \\
\hline Elastic constant $C_{T}$ & $N / m^{2}$ & $0.66 \times 10^{11}$ \\
\hline Band gap $E_{g}^{G a N}(0)$ & $e V$ & 3.42 \\
\hline Band gap $E_{g}^{A l N}(0)$ & $e V$ & 6.13 \\
\hline $\begin{array}{l}\text { Drain-gate \& gate-source distance (LGD } \\
\& \text { LGS) }\end{array}$ & $\mu m$ & 8 \\
\hline gate with & $\mu m$ & 100 \\
\hline Gate length & $\mu m$ & 4 \\
\hline AlGaN unintentional doping density & $\mathrm{cm}^{-3}$ & $1 \times 10^{18}$ \\
\hline AlGaN Si doping density & $\mathrm{cm}^{-3}$ & $2 \times 10^{18}$ \\
\hline Electron effective mass $m_{A l_{m} G a_{1-m} N}^{*}$ & $m_{0}$ & $0.252 \mathrm{~m}+0.228$ \\
\hline Static dielectric constant $\varepsilon_{S}\left(A l_{m} G a_{1-m} N\right)$ & $\varepsilon_{0}$ & $-0.3 m+10.4$ \\
\hline Schottky barrier height $\Phi_{B}(m)$ & $e V$ & $1.3 \mathrm{~m}+0.84$ \\
\hline Interface roughness parameter $\mathrm{L}$ & $n m$ & 1.5 \\
\hline Dislocation charge density $N_{\text {diss }}$ & $\mathrm{cm}^{-2}$ & $1 \times 10^{10}$ \\
\hline Lattice constant $\mathrm{c}$ & $A^{\circ}$ & 5.185 \\
\hline Average displacement of the interface & $n m$ & 1 \\
\hline Auto-correction length of $\Delta$ & $n m$ & 7.5 \\
\hline
\end{tabular}

Table 2- Specific polarization charge values for the PCF scattering potential (Refs. 10, 26)

\begin{tabular}{|c|c|c|c|c|c|}
\hline$V_{G S}(V)$ & $\rho_{0}\left(10^{-2} C / m^{2}\right)$ & $\rho_{1}\left(10^{-2} \mathrm{C} / \mathrm{m}^{2}\right)$ & $\rho_{2}\left(10^{-2} C / m^{2}\right)$ & $\rho_{3}\left(10^{-2} \mathrm{C} / \mathrm{m}^{2}\right)$ & $\rho_{4}\left(10^{-2} C / m^{2}\right)$ \\
\hline 0.6 & & & 1.451 & 1.463 & 1.453 \\
\hline 0.7 & & & 1.450 & 1465 & 1.453 \\
\hline 0.8 & & & 1.450 & 1.466 & 1.452 \\
\hline 0.9 & 1.455 & 0 & 1.449 & 1.467 & 1.452 \\
\hline 1.0 & & & 1.448 & 1.469 & 1.452 \\
\hline 1.1 & & & 1.447 & 1.470 & 1.451 \\
\hline
\end{tabular}




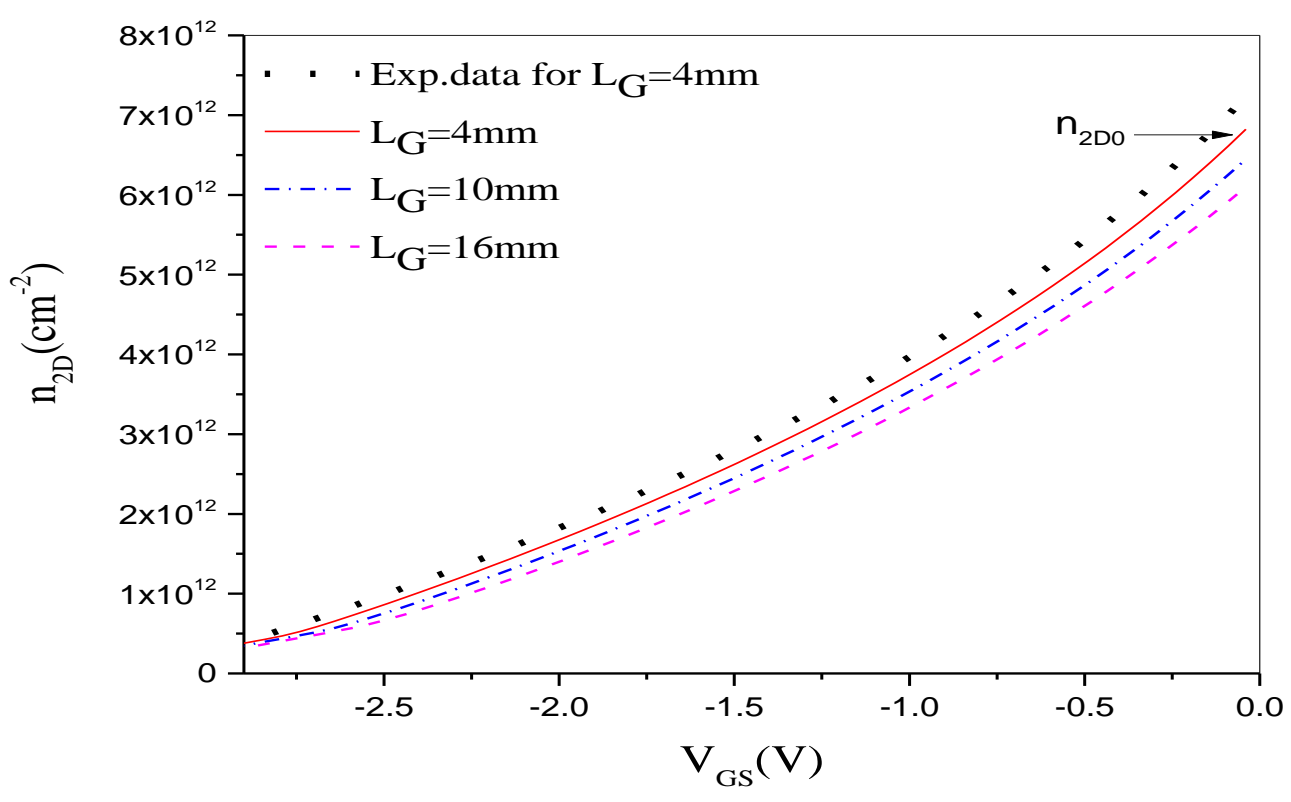

Fig. 2 - The 2DEG density ( $\left.n_{2 D}\right)$ verse $V_{G S}$ for $A l_{0.21} G a_{0.79} N / G a N$ HEMTs with different gate lengths in comparison with experimental data [31].

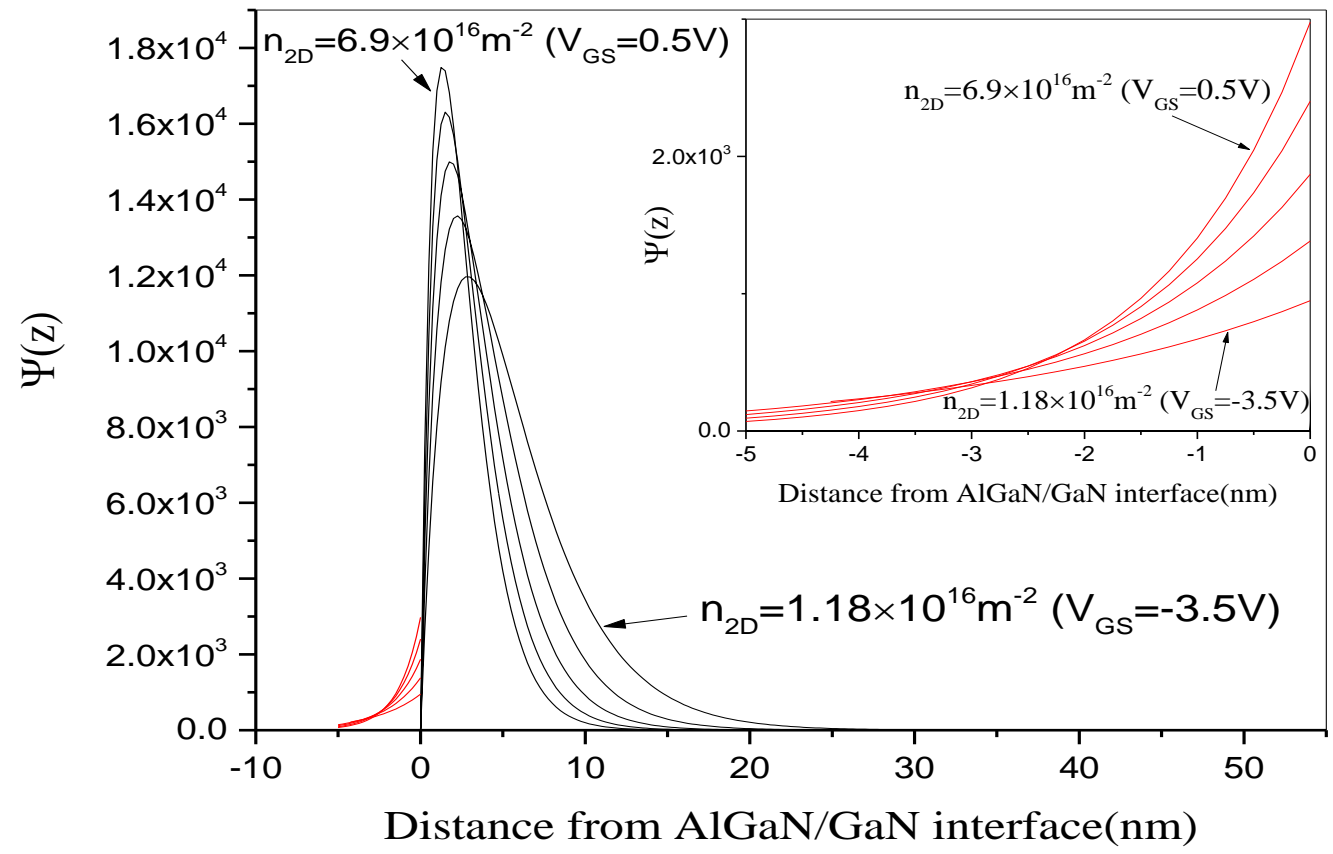

Fig. 3 - The electron wave function $\psi(z)$ as a function of the distance from $A l_{0.21} G a_{0.79} N / G a N$ interface under different $n_{2 D}$ (here $n_{2 D}$ corresponds to the electron density under the gate region as a function of gate bias). 


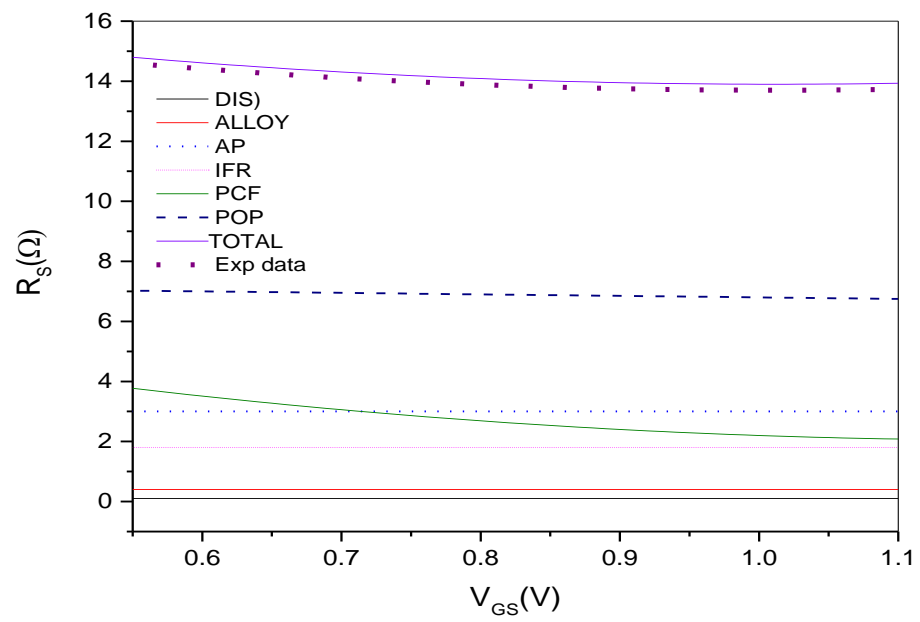

Fig. 4 - Calculated specific $R_{S}$ as a function of the forward gate-source voltage for PCF, POP, AP, IFR, ALLOY and DIS scatterings as well as the total $R_{S}$ (TOTAL) and the measured $R_{S}$ values (the MEASURED) at room temperature in comparison with experimental data [10].

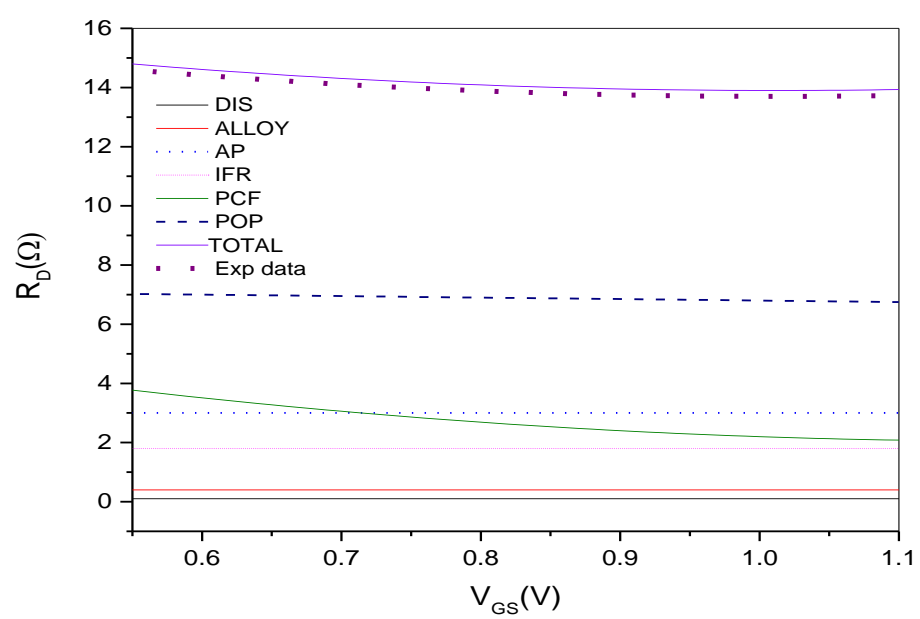

Fig. 5 - Calculated specific $R_{D}$ as a function of the forward gate-source voltage for PCF, POP, AP, IFR, ALLOY and DIS scatterings as well as the total $R_{D}$ (TOTAL) and the measured $R_{S}$ values (the MEASURED) at room temperature in comparison with experimental data [10]. 


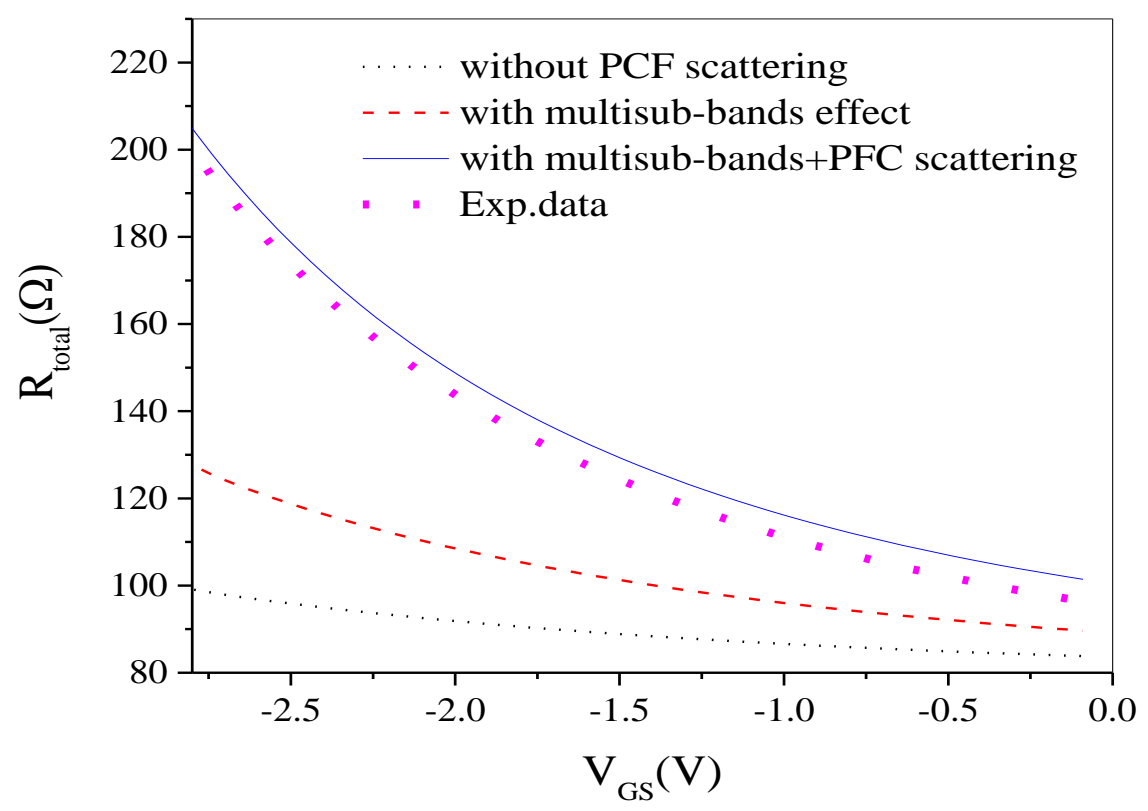

Fig. 5 - Source-drains channel resistance without PCF scattering effect (dot line), with a multi sub-band effect (dashed line) and with all effect (solid line) in comparison with experimental data for $A l_{0.21} G a_{0.79} N / G a N$ HEMTs [31].

\section{Conclusions}

In this paper, an accurate numerical model for source-drain channel resistance has been developed for the $\mathrm{AlGaN} / \mathrm{GaN}$ based HEMTs. This model is able to accurately predict the dependence of source-drain channel resistance on the PCF scattering, multi sub-band. Calculated specific $R_{S}$ and $R_{D}$ as a function of forward gate-source voltage and PCF scattering and multi sub-band effect are not ignorable in source-drain channel resistance AlGaN/GaN HEMTs. According to the numerical calculations, the effect of multi sub-band and PCF scattering on the increase of sourcedrain channel resistance is $35 \%$ and $65 \%$, respectively, with the effect of PCF being almost twice as high as multi subband. If most electrons are in the first sub-band, the effect of the multi sub-band will be 5\% and PCF \% 95, and the PCF scattering will have the greatest effect on total resistance.

\section{Acknowledgment}

We are a faculty member of Islamic Azad University, Khoy Branch, and all resources are provided to our work through the University. The authors would like to thank Khoy Branch.

\section{References}

[1] A. Jarndal, et al., (2019). Reliable noise modeling of GaN HEMTs for designing low - noise amplifiers. Int J Numer Model. 2585,1-13.

[2] Ma, J. \& Matioli, E. Slanted, (2017). Slanted tri-gates for high-voltage GaN power devices, IEEE Electron Device Lett. 38, 1305-1308.

[3] G Tang, et al, (2017). Digital integrated circuits on an E-mode GaN power HEMT platform, IEEE Electron Device Lett. 38, 1282-1285.

[4] M Blaho, et al, (2017). Annealing, temperature, and bias-induced threshold voltage instabilities in integrated E/D-mode InAlN/GaN MOS HEMTs, Appl. Phys. Lett. 111, 033506. 
[5] K Zhang, et al, (2017). High-Linearity AlGaN/GaN FinFETs for Microwave Power Applications, IEEE Electron Device Lett. 38, 615-618.

[6] H Chiu, et al, (2017). RF Performance of in Situ SiNx Gate Dielectric AlGaN/GaN MISHEMT on 6-in Silicon-On-Insulator Substrate, IEEE Trans. Electron Devices. 64, 4065-4070.

[7] S Sun, et al, (2016). AlGaN/GaN metal-insulator-semiconductor high electron mobility transistors with reduced leakage current and enhanced breakdown voltage using aluminum ion implantation, Appl. Phys. Lett. $108,013507$.

[8] D. F Brown, et al, (2017). Self-Aligned AlGaN/GaN FinFETs, IEEE Electron Device Lett. 38, 1445-1448.

[9] L. Yang, et al, (2017). Enhanced gm and fT with High Johnson's Figure-of-Merit in Tin Barrier AlGaN/GaN HEMTs by TiN-Based Source Contact Ledge, IEEE Electron Device Lett. 38, 1563-1566.

[10] P.Cui, et al, (2017). Influence of different gate biases and gate lengths on parasitic source access resistance in $\mathrm{AlGaN} / \mathrm{GaN}$ heterostructure FETs, IEEE Trans. Electron Devices. 64, 1038-1044.

[11] P. Cui, et al, (2017). Effect of polarization Coulomb field scattering on device linearity in AlGaN/GaN heterostructure field-effect transistors, J. Appl. Phys. 122, 124508.

[12] A. Douara, et al, (2018). Optimization of two - dimensional electron gas characteristics of AlGaN/GaN high electron mobility transistors, Int J Numer Model. 2518, 1-9.

[13] R Yahyazadeh., A Asgari A. M Kalafi, (2006). Effect of depletion layer on negative differential conductivity in AlGaN/GaN high electron mobility transistor, Physica E, 33, 77-82.

[14] A. Asgari, S. Babanejad, L. Faraone. (2011). Electron mobility, Hall scattering factor and sheet conductivity in AlGaN/AlN/GaN heterostructures, J. Appl. Phys, 111, 113713.

[15] V. Fiorentini, F. Brenardini, O. Ambacher, (2002). Evidence for nonlinear macroscopic polarization in III-V nitride alloy heterostructures, J. Appl. Phys. 80, 1204.

[16] R. yahyazadeh, (2018). Analytical-numerical model for sheet resistance of high electron mobility transistors, Journal of Non - Oxide Glasses, 10, 2, 57 - 63.

[17] S Kabra, H Kaur, S Haldar, M Gupta, R.S. Gupta, (2008). An analytical drain current model for graded channel cylindrical/surrounding gate MOSFET, Solid-State Electron, 52, 25.

[18] A. S. Kumar, N. S. Garigapati, D. Sahaa, (2019). Low field mobility in electrostatically evolved AlGaN/GaN one-dimensional channel from a two-dimensional electron gas system, J. Appl. Phys. 115, 032105.

[19] P. Roblin, H. Rahdin, (2002) High-speed Heterostructure Devices from Device Concepts to Circuit Modeling, Cambridge University Press, Cambridge, 277.

[20] M.A. Huque, S.A.E, liza, T.Rahman, S.K.Islam, (2009). Temperature dependent analytical model for currentvoltage characteristics of $\mathrm{AlGaN} / \mathrm{GaN}$ power HEMT, Solid State Electronics, 53, 3, 341-348.

[21] N.S.Swamy, A.K.Datta, (2018). Analytical Models for the 2DEG Density, AlGaN Layer Carrier Density, and Drain Current for AlGaN/GaN HEMTs, IEEE Transactions on Electron Devices. 65, 936-944.

[22] Z. Hashempour, et al, (2009). Numerical performance evaluation of AlGaN/GaN high electron mobility transistors including gate length effects, Physica E, 41, 1517-1521.

[23] M.Yang, et al, (2018).Determination of the polarization and strain distribution in AlGaN/GaN heterostructure field-effect transistors, J. Phys. Chem. Solids, 123, 223-227.

[24] M. N. Gurusinghe, S. K. Davidsson, and T. G. Andersson, (2005). Two-dimensional electron mobility limitation mechanisms in Alx Ga1-x N/GaN heterostructures Phys. Rev. B, 72, 4, 045316.

[25] Z.W. Zhou, J.-Xun Sun, M. A. Khan, (2015). Shifted Fang-Howard wavefunction for the two-dimensional electron gas and application to MOSFETs, Int. J. Mod. Phys B.vol 29, 1450250.

[26] M. Yang et al, (2016). Effect of polarization Coulomb field scattering on parasitic source access resistance and extrinsic transconductance in AlGaN/GaN heterostructure FETs, IEEE Trans. Electron Devices, 63, 4, 14711477.

[27] L. Hsu and W. Walukiewicz, (2001). Effect of polarization fields on transport properties in AlGaN/GaN heterostructures, J. Appl. Phys, 89, 1783.

[28] T. H Yu, K F. Brennan, (2001).Theoretical study of the two-dimensional electron mobility in strained IIInitride heterostructures, J. Appl. Phys, 89, 3827.

[29] R.yahyazadeh, (2014). Effect of Temperature on the Total Mobility of AlGaN/GaN High Electron Mobility Transistors ECS Trans, 60, 1,1051-1055.

[30] J. C. Freeman, (2004). channel temperature model for microwave AlGaN/GaN power HEMTs on SiC and sapphire, IEEE MTT-S Int. Microwave Symp. Dig. 3, 2031.

[31] P. Cui, et al, (2018). Effect of different gate lengths on polarization Coulomb field scattering potential in $\mathrm{AlGaN} / \mathrm{GaN}$ heterostructure field-effect transistors, Science Reports, 8, 9036. 